\title{
Unresectable Advanced Gastric Cancer with Skin Invasion followed by Total Gastrectomy after Second-Line Chemotherapy
}

\author{
Hidenori Maki Yasuhiro Yuasa Satoshi Fujiwara Mizuki Fukuta \\ Taihei Takeuchi Keisuke Fujimoto Takao Tsuneki Yuta Matsuo \\ Osamu Mori Shohei Eto Atsushi Tomibayashi Hisashi Ishikura \\ Department of Surgery, Tokushima Red Cross Hospital, Komatsushima City, Japan
}

\section{Keywords}

Unresectable gastric cancer - Ramucirumab/Paclitaxel · Conversion surgery

\begin{abstract}
Conversion surgery has been reported but few cases have undergone surgical R0 resection after second-line chemotherapy. We report a case of an unresectable locally advanced gastric cancer in a patient who finally underwent the operation (R0) after second-line chemotherapy. The 77-year-old male was diagnosed with gastric cancer (cT4 [SI; Skin, Liver] NOM0 c Stage IIIA) with invasion to the skin of the abdominal wall, and chemotherapy was initially performed because of his poor performance status and due to the large defect in the abdominal wall that might occur if an operation was performed. Partial response (PR) was observed after $\mathrm{S}$ $1+$ CDDP (SP) therapy, which was then stopped after which progressive disease (PD) was observed. Ramucirumab+Paclitaxel (RAM/PTX) therapy was chosen as second-line therapy, and PR was obtained again, following which total gastrectomy was performed (D2 dissection of
\end{abstract}


lymph nodes, Roux-en-Y reconstruction, and combined resection of the partial skin and the affected region of the liver). At 30 months postoperatively, no recurrence has occurred and the patient is alive after the operation without chemotherapy.

\section{Introduction}

Recently, some reports have indicated the utility of the conversion surgery for locally advanced gastric cancer. However, this subject has remained controversial with only a few reports about R0 resection in patients after second-line chemotherapy or operation after using Ramucirumab, an anti-Vascular Endothelial Growth Factor Receptor-2 (VEGFR-2) antibody $[3,4]$. We report a case of a patient who underwent R0 resection after Ramucirumab+ Paclitaxel (RAM/PTX) as second-line therapy.

\section{Case Presentation}

\section{Patient and Methods}

A 77-year-old man presented with a complaint of a lump on the upper abdominal wall for several weeks. His performance status (PS) was Grade 2 (Height, $168 \mathrm{~cm}$; Weight, $58 \mathrm{~kg}$; BMI, $20 \mathrm{~kg} / \mathrm{m}^{2}$ ). He had a history of appendicitis that was operated upon. On physical examination, the tumor was located on the upper left side of the abdominal wall measuring around $5 \mathrm{~cm}$ with poor mobility and partial ulceration but without pain (Fig. 1). At a nearby hospital, he had previously undergone physical examination and computed tomography (CT) for his symptoms. Advanced gastric cancer with invasion to the skin of the abdominal wall was suspected and, for more specific investigation and therapy, he was referred to our department. We obtained informed consent from all participants, and all procedures therapy were performed in accordance with the Declaration of Helsinki.

\section{Laboratory Data}

His blood investigation revealed the following: hemoglobin $8.7 \mathrm{~g} / \mathrm{dL}$, white blood cell count 6,060 $/ \mu \mathrm{L}$, platelets $321,000 / \mu \mathrm{L}$, prothrombin time-international nomarized ratio (PTINR) 1.05, sodium $140 \mathrm{mmol} / \mathrm{L}$, potassium $4.2 \mathrm{mmol} / \mathrm{L}$, chlorine $105 \mathrm{mmol} / \mathrm{L}$, creatinine 0.91 $\mathrm{mg} / \mathrm{dL}$, aspartate aminotransferase (AST) $13 \mathrm{U} / \mathrm{L}$, alanine aminotransferase (ALT) $10 \mathrm{U} / \mathrm{L}$, alkaline phosphatase $249 \mathrm{U} / \mathrm{L}, \gamma$-guanosine triphosphate cyclohydrolase $(\gamma$-GTP) $14 \mathrm{U} / \mathrm{L}$, LD $156 \mathrm{U} / \mathrm{L}$, total bilirubin $0.8 \mathrm{mg} / \mathrm{dL}$, C-reactive protein $0.41 \mathrm{mg} / \mathrm{dL}$, carcinoembryonic antigen$\mathrm{S}$ (CEA-S) $1.6 \mathrm{ng} / \mathrm{mL}$, alpha-fetoprotein $1.83 \mathrm{ng} / \mathrm{mL}$ and carbohydorate antigen 19-9 (CA 199) $<2 \mathrm{U} / \mathrm{mL}$. There was moderate anemia but there was no organ failure and or elevation of tumor marker.

\section{Upper Gastrointestinal Endoscopy}

On Upper gastrointestinal endoscopy, a Type 3 tumor that measured around $40 \mathrm{~mm}$ was observed occupying from the angle of the stomach to the anterior wall of the antrum of the stomach (Fig. 2). 


\section{Case Reports in Oncology}

Biopsy

On biopsy, the histological type was revealed as tubular adenocarcinoma (tub1) and immunostaining for HER2 was negative.

\section{Thoracoabdominal Enhanced CT}

Thoracoabdominal enhanced CT revealed invasion of the tumor from the anterior wall of the stomach to the lateral region of the liver and infiltration from the abdominal wall to the skin. There was no evidence of metastasis or ascites (Fig. 3).

\section{Therapeutic Course}

On the basis of these findings, we diagnosed advanced gastric cancer (L-Less, Gre, type 3, $40 \mathrm{~mm}$, tub1), cT4b (SI; liver, skin) N0M0 c Stage IIIA. Initially we anticipated chemotherapy considering that the patient's PS was Grade 2 and the defect of abdominal wall was too large for surgery. In addition, the patient did not want to undergo surgery. S-1+Cisplatin (SP) therapy was performed as first-line therapy every 3 weeks [1].

Obvious shrinkage of the tumor invasion to the skin was observed, and we determined that PR was obtained at the end-point of 4th course of SP therapy. However, because of malaise (Grade 2), we had to set a 1-month interval. During the interval, the invasion to the skin gradually increased again; hence, another two courses of SP regimen were added with decreased dose. After six courses of SP therapy had been completed, PR was obtained again by the re-shrinkage of the skin tumor on physical examination and upper gastrointestinal endoscopy and CT (Fig. 4, 5). At this point, the patient was not amenable to surgery or chemotherapy; hence, we adopted a "wait-and-see" approach. During this period, the skin tumor underwent re-growth gradually for which RAM/PTX therapy (3-week administration at 1-week intervals) was introduced as the second line chemotherapy, 2 months after the previous one [2]. Over the course of therapy, anemia (Grade 3) and neutropenia (Grade 2) were observed and we were compelled to skip one administration each of PTX in course 2 and 3 but RAM dosage was completely administrated through all five courses (Fig. 6). Throughout the second line of therapy, the skin tumor kept decreasing visually and enhanced abdominal CT revealed the shrinkage of the primary tumor and the invasion to the lateral area of the liver and skin. Similar findings were observed on gastrointestinal endoscopy; and hence, we determined that PR was obtained again (Fig. 7). Since his general condition was observed have improved, we planned to perform surgery with the aim of reaching a chemotherapy-free status.

\section{Surgical Findings}

We performed open total gastrectomy with D2 lymph nodes dissection (Roux-en-Y reconstruction) with combined partial resection of the skin, abdominal wall, and liver. There were no findings of the peritoneal dissemination, metastasis to the liver, or ascites (Fig. 8). The components separation method was performed for abdominal wall closure with the defect of the skin by exposure of the anterior sheath of abdominal rectal muscles and resection of partial aponeurosis of the right external abdominal oblique muscle. 


\section{Case Reports in Oncology}

Case Rep Oncol 2019;12:671-680

DOI: $10.1159 / 000502415$

(c)

2019 The Author(s). Published by S. Karger AG, Base www.karger.com/cro

Maki et al.: Unresectable Advanced Gastric Cancer with Skin Invasion followed by Total Gastrectomy after Second-Line Chemotherapy

\section{Results}

Postoperatively, the final pathological analysis was finally ypT4b (SI:skin), int, INFc, ly0, v0, pPM0, pDM0, ypN0, yp Stage III, R0 -resection, and the histological treatment effectiveness was Grade 1a. Dehiscence of the defect of the skin was observed after the operation, and the skin of the right inguinal area was grafted by the Department of Plastic Surgery 1 month after postoperatively. Considering the final pathological findings, we recommended post-operative adjuvant chemotherapy; however the patient declined this treatment. Over the regular followups for 30 months after his first therapy, there has been no evidence of the recurrence, and the patient is alive without chemotherapy.

\section{Discussion}

SP therapy has already been standardized as a first-line chemotherapy course for unresectable advanced gastric cancer. The efficacy of RAM/PTX therapy as the second-line has been demonstrated in the RAINBOW trial [2]. Recently, immunity check point inhibitors have been introduced as a novel therapeutic choice for unresectable advanced gastric cancer. Previously, the long-term survival of unresectable advanced gastric cancer has been known to be poor; however, some rare cases have been reported that achieved long-term survival or underwent conversion surgery after effective first- or second-line chemotherapy even though they were originally diagnosed as unresectable at first $[3,4]$. This indicates that the possibility of the curative effects of chemotherapy alone as well as a combination of surgery. However, the timing of introduction and the long-term prognosis of neo- adjuvant chemotherapy (NAC) or conversion surgery has remained unclear so far, and the protocols for surgery depend on each individual institute $[3,4]$. The median survival time (MST) has increased to 12-16 months $[2,4]$, but the long-term prognosis of recurrent or metastatic cases remains poor because of rapid progress. In the Gastric Cancer-Treatment Guidelines (15th edition; Japanese Gastric Cancer Association), it is clearly mentioned that all six currently available chemotherapies involving Nivolumab should be considered for unresectable advanced gastric cancer after third-line chemotherapy [5]. In our case, we diagnosed stage III gastric cancer with direct invasion to the liver, abdominal wall, and skin. Considering the patient's PS and aversion to undergo surgery, if surgical therapy had been chosen initially, completing R0 resection might have been difficult; there was high surgical invasion and the loss of quality of life because of the defect of the abdominal wall. Therefore, we chose the chemotherapy at first and finally achieved R0 resection after administering RAM/PTX therapy as a second-line therapy.

We searched literatures in the Japanese data base "Ichuushi" with the key words "gastric cancer" and "conversion" during last 5 years. Though several reports were available, case reports about conversion surgery after second-line chemotherapy are very rare and, additionally, there are only five cases involving our own that underwent RAM/PTX as second-line therapy (Table 1). This indicates it is rare to get conversion surgery after second-line chemotherapy and that there might be tendency to avoid using Ramucirumab if conversion surgery is planned from a standpoint of its anti-angiogenic effect and wound healing. Each reported case underwent conversion surgery at the point of obtaining PR or CR with initially diagnosed unresectable factors but no reports are available on locally advanced gastric cancer with direct 
invasion to the skin which has undergone surgical resection after a chemotherapy. Thus, our case may be unique.

In the discussion regarding the choice to administer chemotherapy before operation, according to the attached instruction, the frequency of the complication associated with wound healing of RAM (anti-VEGFR-2 antibody) is about $0.6 \%$. The average half reduction time of RAM/PTX in the condition of repeated administration is reported to be approximately 181$218 \mathrm{~h}$, and an interval of more than 28 days after the last administration is suggested before an operation [6]. After final administration, we kept an adequate interval (37 days) before the operation. Skin grafting was performed for the defect of the skin but we consider that the postoperative dehiscence of the wound was mainly caused by the size of the defect and not by poor grafting. It might be permissive to re-challenge SP therapy as the second-line therapy. In a recent report, the MST of selected patients with stage IV advanced gastric cancer who had good response for chemotherapy followed by surgical therapy is significantly better than that of patients who underwent chemotherapy alone. In particular, they reported that if R0 resection is possible, the MST (41.3 months) is much better than that of R1 or R2 resection 21.2 months) $[3,7,8]$. In this case, we started the treatment without the aim to perform conversion surgery. Nonetheless, this case demonstrates the usefulness of the introduction of the chemotherapy for cases initially diagnosed as unresectable advanced cancer with the goal of R0 resection. Moreover, RAM/PTX therapy before the surgical therapy for such cases is clinically safe. Thus, we consider it clinically significant.

\section{Acknowledgements}

We would like to thank Editage (www.editage.com) for English language editing.

\section{Statement of Ethics}

This case report was performed under the Declaration of Helsinki. Ethical approval has been exempted by our institution because this is a case report.

\section{Disclosure Statement}

We have no financial conflicts of interest to disclose concerning this case report.

\section{Funding Sources}

This research received no specific grant from any funding agency in the public, commercial, or not-for-profit sectors. 


\section{Author Contributions}

All author helped to draft the manuscript. All authors read and approved the final manuscript.

\section{References}

1 Ryu MH, Baba E, Lee KH, Park YI, Boku N, Hyodo I, et al.; SOS study investigators. Comparison of two different S-1 plus cisplatin dosing schedules as first-line chemotherapy for metastatic and/or recurrent gastric cancer: a multicenter, randomized phase III trial (SOS). Ann Oncol. 2015 Oct;26(10):2097-101.

2 Wilke H, Muro K, Van Cutsem E, Oh SC, Bodoky G, Shimada Y, et al.; RAINBOW Study Group. Ramucirumab plus paclitaxel versus placebo plus paclitaxel in patients with previously treated advanced gastric or gastrooesophageal junction adenocarcinoma (RAINBOW): a double-blind, randomised phase 3 trial. Lancet Oncol. 2014 Oct;15(11):1224-35.

3 Yamaguchi K, Yoshida K, Tanahashi T, Takahashi T, Matsuhashi N, Tanaka Y, et al. The long-term survival of stage IV gastric cancer patients with conversion therapy. Gastric Cancer. 2018 Mar 21(2):315-23.

4 Fukuchi M, Mochiki E, Ishiguro T, Kumagai Y, Ishibashi K, Ishida H. Prognostic significance of conversion surgery following first or second-line chemotherapy for unresectable gastoric cancer. Anticancer Res. 2018 Nov;38(11):6473-8.

5 The Gastric Cancer Treatment Guidelines. 15th ed. Japanese Gastric Cancer Association.

6 Proper usage guide of Ramucirumab QANo.RAM07900. Available from: http://www.lillymedical.jp/jp/ja/oncology/cyramza/index.aspx.

7 Shen L, Shan YS, Hu HM, Price TJ, Sirohi B, Yeh KH, et al. Management of gastric cancer in Asia: resourcestratified guidelines. Lancet Oncol. 2013 Nov;14(12):e535-47.

8 Lordick F, Siewert JR. Recent advances in multimodal treatment for gastric cancer: a review. Gastric Cancer. 2005;8(2):78-85.

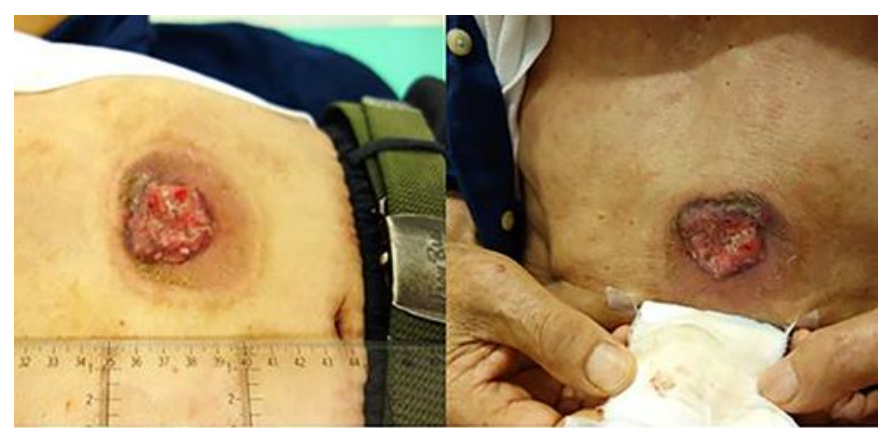

Fig. 1. Tumor invasion to skin of abdominal wall. 


\section{Case Reports in Oncology}

www.karger.com/cro

Maki et al.: Unresectable Advanced Gastric Cancer with Skin Invasion followed by Total Gastrectomy after Second-Line Chemotherapy

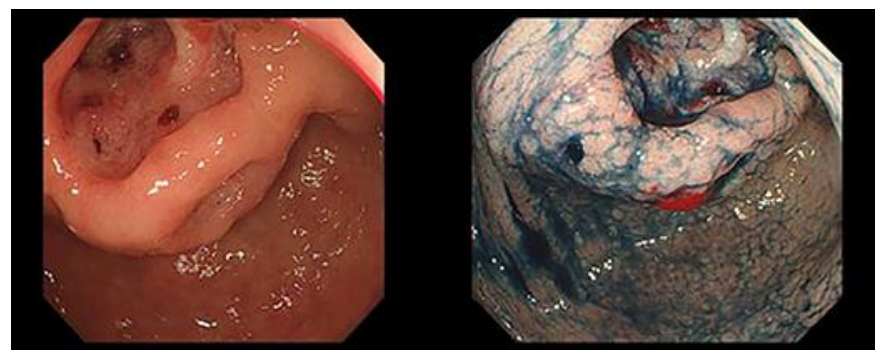

Fig. 2. Upper gastrointestinal endoscopy demonstrated a massive submucosal tumor like tumor measuring $40 \mathrm{~mm}$ over the antrum from the gastric angle.

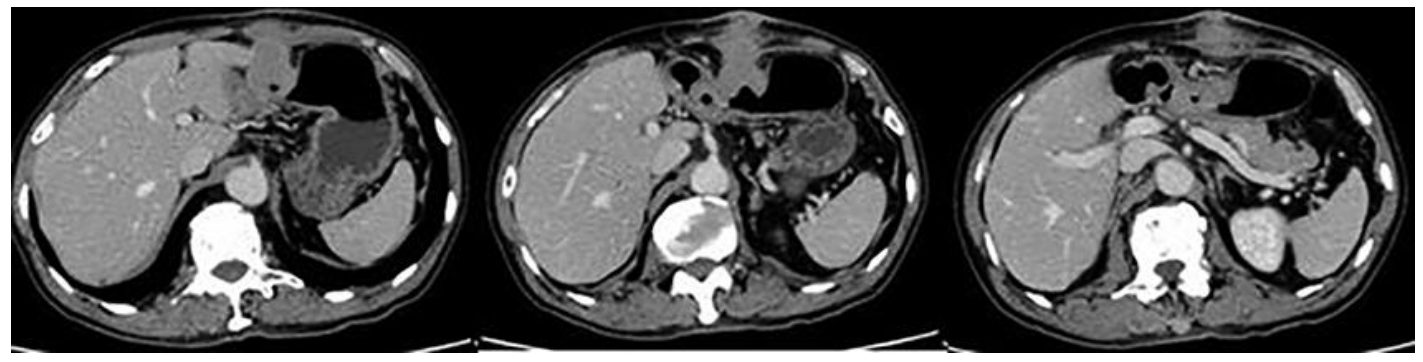

Fig. 3. Computed tomography showing the invasion of the tumor from the anterior wall of the stomach to lateral aspect of the liver and infiltration from the abdominal wall to the skin. 


\section{Case Reports in Oncology}

Case Rep Oncol 2019;12:671-680

DOI: $10.1159 / 000502415$

(C) 2019 The Author(s). Published by S. Karger AG, Basel www.karger.com/cro

Maki et al.: Unresectable Advanced Gastric Cancer with Skin Invasion followed by Total Gastrectomy after Second-Line Chemotherapy

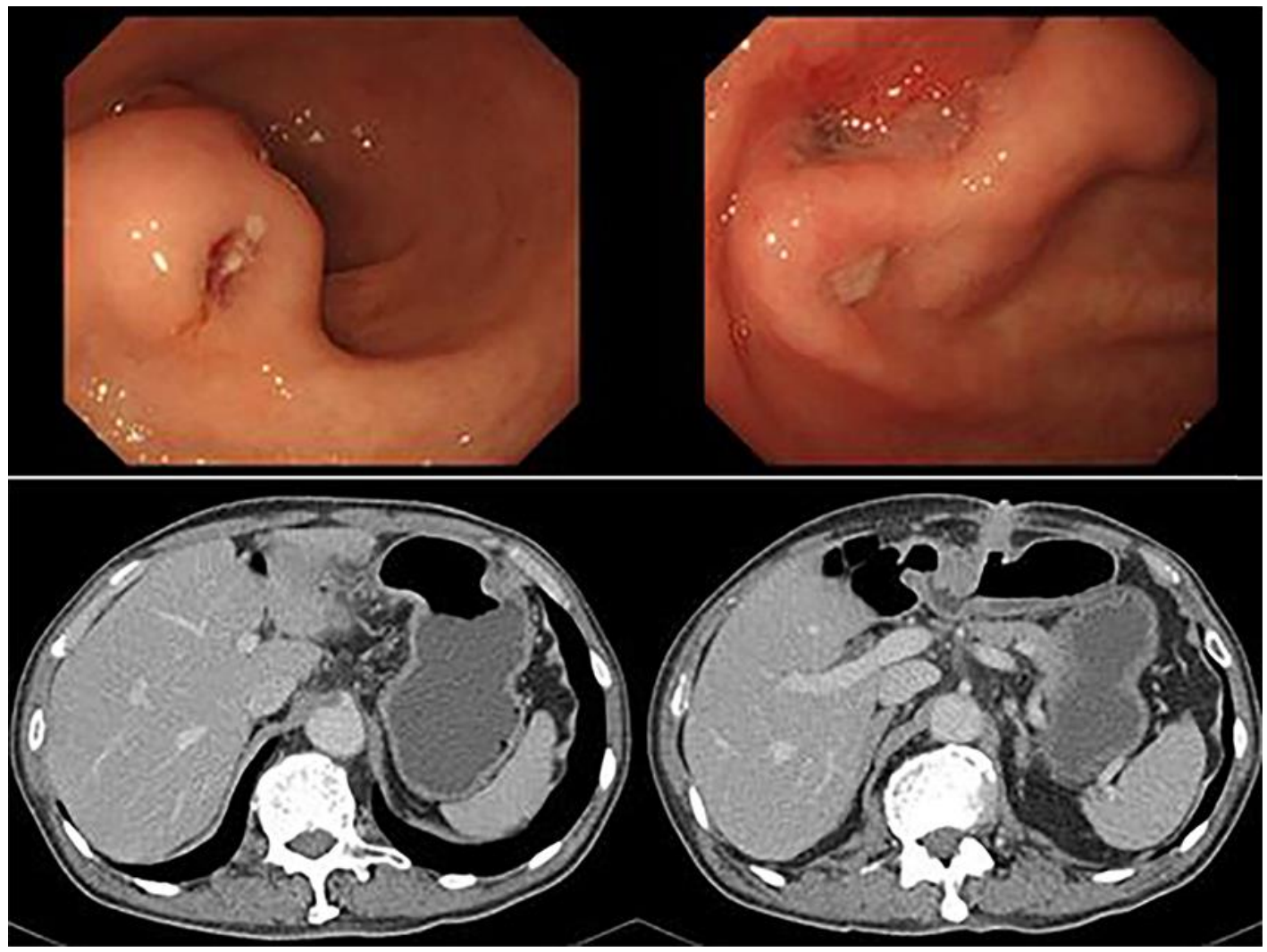

Fig. 4. The tumor of the abdominal wall had clearly reduced in size (Partial response).

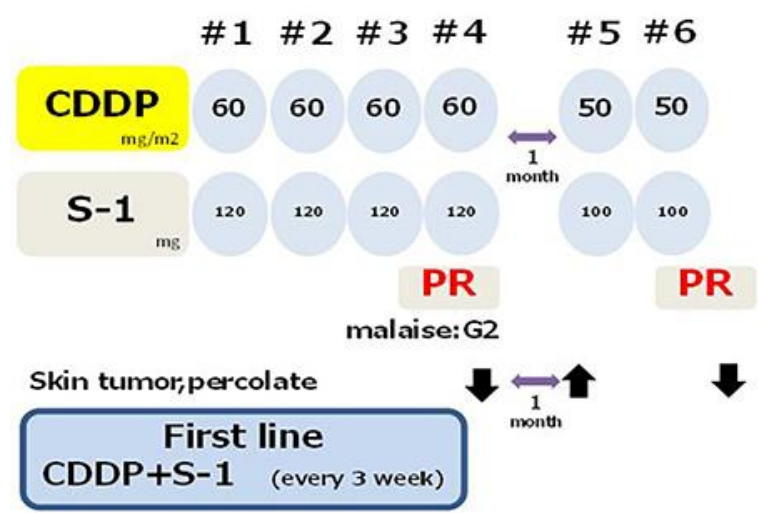

Fig. 5. SP therapy $\left(60 \mathrm{mg} / \mathrm{m}^{2} \mathrm{CDDP}+120 \mathrm{mg} \mathrm{S}-1\right)$ was performed as first-line chemotherapy every three week, after one month of interval the dose was reduced (50 mg/m² CDDP+ $100 \mathrm{mg} \mathrm{S}-1$ ) due to malaise (Grade 2). 


\section{Case Reports in Oncology}

\begin{tabular}{l|l}
\hline Case Rep Oncol 2019;12:671-680 \\
\hline DOI: 10.1159/000502415 & $\begin{array}{l}\text { (c) 2019 The Author(s). Published by S. Karger AG, Basel } \\
\text { www.karger.com/cro }\end{array}$ \\
\hline
\end{tabular}

Maki et al.: Unresectable Advanced Gastric Cancer with Skin Invasion followed by Total Gastrectomy after Second-Line Chemotherapy

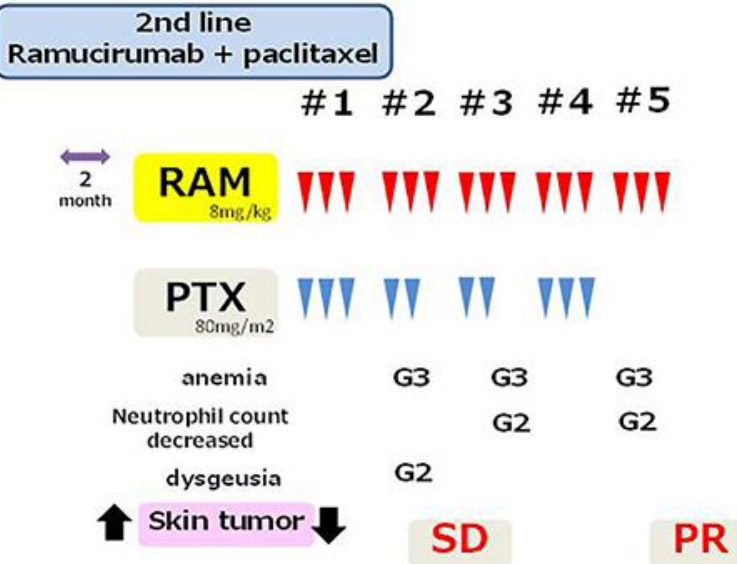

Fig. 6. RAM/PTX therapy ( $\left.8 \mathrm{mg} / \mathrm{m}^{2} \mathrm{RAM}+80 \mathrm{mg} / \mathrm{m}^{2} \mathrm{PTX}\right)$ was started as second-line chemotherapy 1 month after first-line one and PR was obtained again.

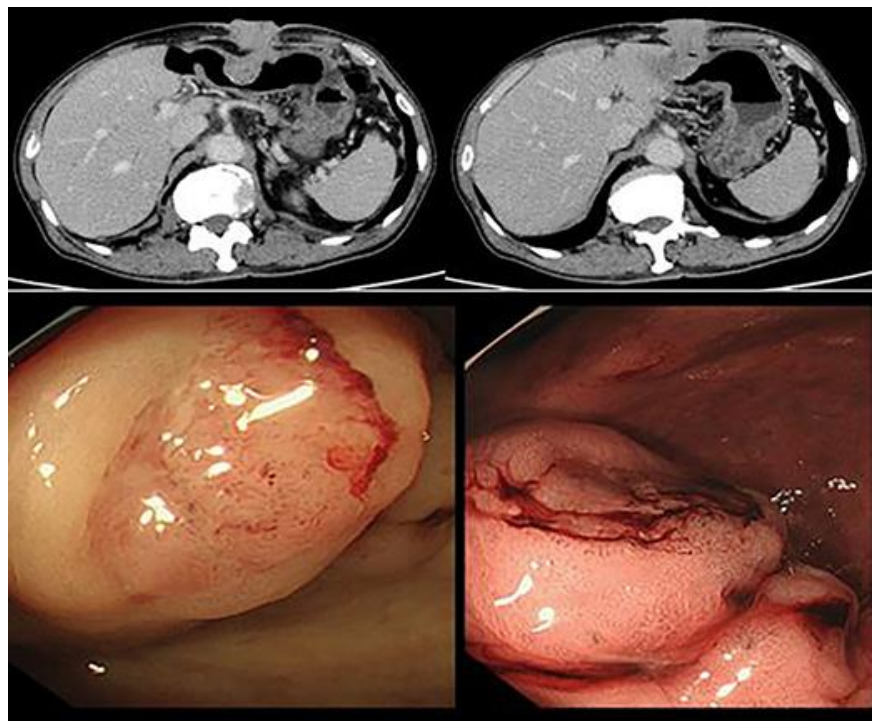

Fig. 7. Computed tomography and gastrointestinal endoscopy: partial response (PR) was obtained again after completed second-line chemotherapy. 


\section{Case Reports in Oncology}

\begin{tabular}{l|l}
\hline Case Rep Oncol 2019;12:671-680 \\
\hline DOI: 10.1159/000502415 & $\begin{array}{l}\text { ( 2019 The Author(s). Published by S. Karger AG, Basel } \\
\text { www.karger.com/cro }\end{array}$ \\
\hline
\end{tabular}

Maki et al.: Unresectable Advanced Gastric Cancer with Skin Invasion followed by Total Gastrectomy after Second-Line Chemotherapy

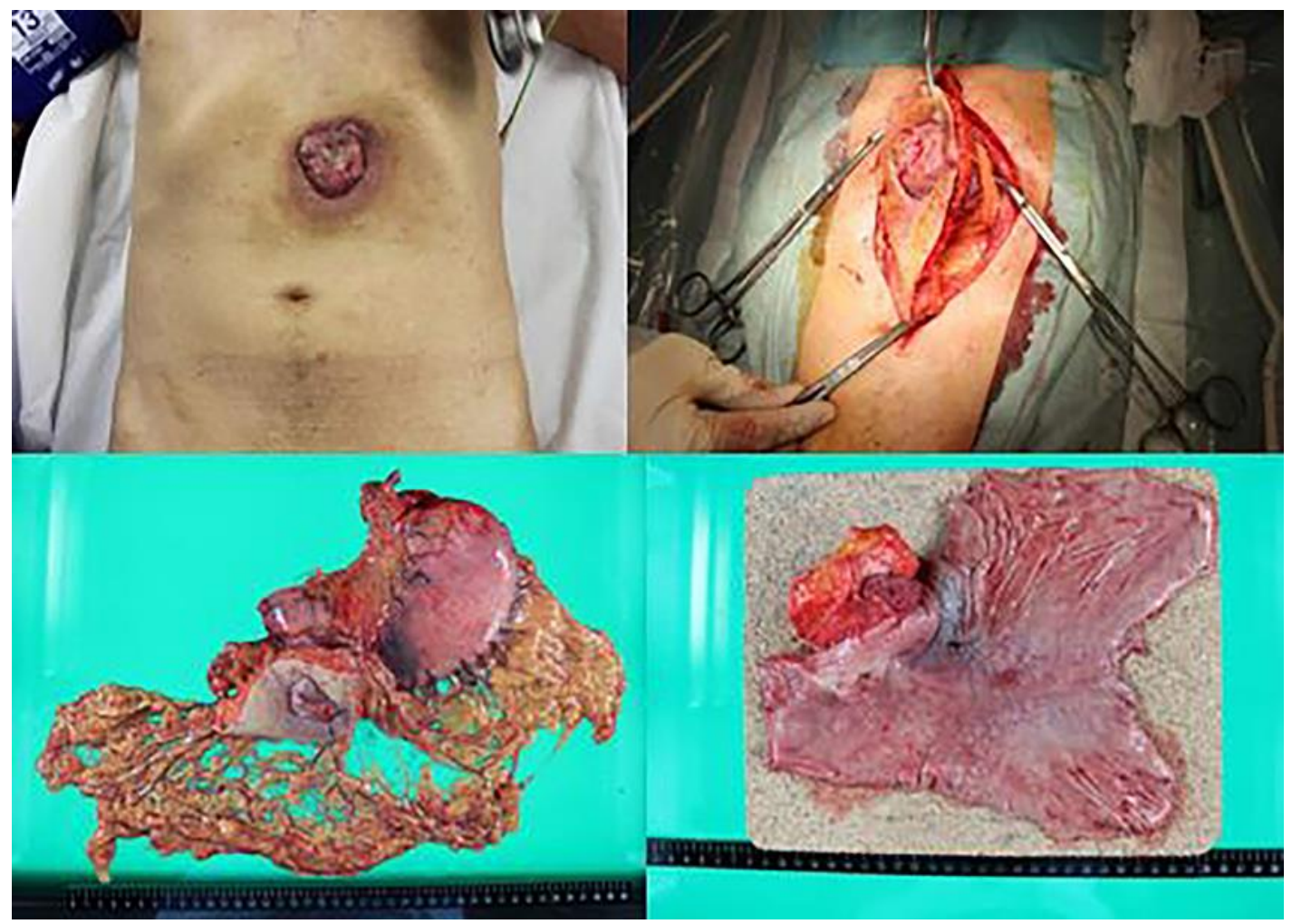

Fig. 8. Surgical findings and the resected stomach.

Table 1. Conversion cases after RAM/PTX as second-line chemotherapy

\begin{tabular}{llllll}
\hline $\begin{array}{l}\text { Age, } \\
\text { years }\end{array}$ & Sex & Unresectable factor & 1st line & 2nd line & Reference \\
\hline 62 & M & Paraaortic lymph node metastasis & S-1/CDDP & RAM/PTX & Hanzawa et al. ${ }^{1}$ \\
63 & M & Liver metastasis & S-1/CDDP & RAM/PTX & Tastubayashi et al. ${ }^{2}$ \\
68 & M & Paraaortic lymph node metastasis & G-SOX & RAM/PTX & Fukuta et al. ${ }^{3}$ \\
$60 \mathrm{~s}$ & M & Invasion to pancreas peritoneal & S-1/CDDP & RAM/PTX & Sakamoto et al. ${ }^{4}$ \\
77 & M & Invasion to skin and liver & S-1/CDDP & RAM/PTX & Our case \\
\hline
\end{tabular}

1 Department of Surgery, Himeji Red Cross Hospital. ${ }^{2}$ Department of Surgery, National Hospital Organization Osaka Minami Medical Center. ${ }^{3}$ Digestive Disease Center (Gastroenterological Surgery), Japan Community Health Care Organization Hokkaido Hospital. 4 Department of Sugery, Wakayama Rosai Hospital. 